Bristol Eye Hospital, Lower Maudlin Street, Bristol BS1 2LX D Papakostopoulos P A Bloom R H B Grey

J C Dean Hart

Correspondence to: Philip A Bloom, Moorfields Eye Hospital, City Road, London ECIV 2PD.

Accepted for publication 21 February 1992

\title{
The electro-oculogram in central retinal vein occlusion
}

\author{
D Papakostopoulos, PA Bloom, RHB Grey, J C Dean Hart
}

\begin{abstract}
As part of a prospective masked study, the electro-oculogram (EOG) was recorded from 28 patients within 48 days of developing central retinal vein occlusion (CRVO). The EOG light peak/dark trough ratio $(\mathrm{Lp} / \mathrm{Dt}) \times 100$ was significantly lower in the affected than in the unaffected eyes of patients $(p<0.001)$, and abnormally low in absolute terms in 20 patients (71\%). All unaffected fellow eyes had a normal EOG ratio. The mean $L p$ amplitude of affected eyes was significantly smaller than that of unaffected eyes $(p<0.001)$, whereas the differences in mean Dt amplitudes between affected and unaffected eyes were not statistically significant. The $L p$ amplitude in the affected eye was $48 \%$ or less of that in the unaffected eye in the eight patients $(29 \%)$ who developed rubeosis iridis during the 9 month follow-up, and in six others. No patient whose Lp amplitude in the affected eye was greater than $48 \%$ of that in the unaffected eye, developed rubeosis. It is concluded that the Lp amplitude is abnormal in patients with acute CRVO. The degree of this abnormality bears a relation to the development of rubeosis, which might prove a useful indicator of whether to institute or withhold panretinal photocoagulation.

(Brf Ophthalmol 1992; 76: 515-519)
\end{abstract}

Central retinal vein occlusion (CVRO) is a common cause of visual loss and may lead to rubeosis iridis and to potentially painful neovascular glaucoma. The risk of this complication may be reduced by panretinal photocoagulation following the early identification of high risk cases. Attempts have been made to predict rubeosis using various parameters but no method of prediction has gained wide acceptance in clinical practice.

Electrophysiological investigations in cases of CRVO have largely been concerned with changes in the electroretinogram..$^{1-7}$ There are only a few contradictory references to electro-oculogram (EOG) changes in CRVO. ${ }^{18-10}$ In his original description of the EOG, Arden described a case of CRVO in which the EOG was severely affected. ${ }^{8}$ Ashworth reported four cases of CRVO, in all of which the EOG was affected to varying degrees. ${ }^{9}$ Babel reported one case whose EOG was unaffected. ${ }^{1}$ It was therefore decided to investigate, prospectively, the EOG changes in acute CRVO.

\section{Patients and methods}

PATIENT SELECTION

Patients found to be suffering from acute CRVO on presentation to the accident and emergency department of Bristol Eye Hospital, were referred to a special clinic for assessment. All patients presented within 3 weeks of noticing a visual disturbance.

The patients had a detailed clinical examination within 2 weeks of presentation. Patients then underwent electro-oculography within 10 days of clinical examination, and at least 7 days after instillation of any topical medication. This ensured that the effect of pupil dilatation did not influence the results.

The electrodiagnostic investigator (DP) was not provided with clinical details except that patients being assessed had recently suffered a CRVO in one or other eye. Patients were followed up clinically once a month by the same investigator (PB) for 9 months. The EOG results were only analysed after this period of follow-up. If rubeosis was noted during follow-up, panretinal photocoagulation (PRP) was performed.

A total of 28 patients ( 17 male, 11 female; age range 48-85 years, mean 67 years, SD 9 years) were included in the study. Two patients who were initially included, declined to undergo the tests. Eight patients developed rubeosis iridis.

\section{CLINICAL EXAMINATION}

At the initial clinic visit and at each subsequent clinical assessment, corrected visual acuity was measured and careful slit-lamp examination performed, including gonioscopy, to detect the earliest sign of rubeosis iridis. This was considered to be the appearance of rubeotic vessels at the pupil margin or in the iridocorneal angle. In this examination, consideration was given to the fact that early rubeosis may be difficult to see in patients with dark irides. If rubeosis was suspected the patient was reviewed weekly until progression of the subtle signs enabled a definite diagnosis to be made.

Pupil sizes were measured under room illumination, but in no patient was anisocoria more than $1 \mathrm{~mm}$. Fundal examination was performed after pupillary dilatation. A clinical diagnosis of CRVO was made by identification of the typical fundal signs of CRVO, namely dilated and tortuous retinal veins, retinal haemorrhages at the posterior pole extending towards the mid- 
periphery in all four quadrants, and unilateral disc swelling in association with a reduction of visual acuity.

\section{INCLUSION AND EXCLUSION CRITERIA}

Only patients with a definite clinical diagnosis of acute CRVO and who underwent clinical assessment and electro-oculography within 60 days of the onset of visual symptoms were included in the results of the study.

Criteria for exclusion from the study were previous significant ophthalmic disease in either eye, previous ophthalmic surgery, the use of topical or systemic medications, and previous photocoagulation.

EOG MEASUREMENT

The technique employed for recording the EOG was based on that of Arden $e t a l^{8}$ and has been described previously. ${ }^{11}$ In summary the subject was seated $1.5 \mathrm{~m}$ in front of the centre of a panel, upon which were mounted eight neon tube bulbs. Two small red bulbs served as fixation points and were positioned either side of the centre of the screen to subtend an angle of $30^{\circ}$ at the patient. Chlorided silver electrodes were attached to the bridge of the nose and to each outer canthus, and the signals were amplified by an EEG machine.

The subject was instructed to perform fast eye movements from one fixation point to the other, at a rate of one per second; a sequence of eight of these movements was performed and recorded every 2 minutes. Six such sequences were performed in darkness after a 20 minute period of light adaptation. Then with the room and screen illuminated another six sequences were recorded, again each separated by 2 minutes. The mean EOG amplitude for each sequence was calculated. Therefore for each subject, 12 values were obtained from the left and 12 from the right eye in each session, from which were determined the minimum EOG amplitude in the dark (dark
Table 1 EOG Lp/Dt ratios of all patients, ranked in ascending order according to the ratio in the affected eye

\begin{tabular}{lll}
\hline & $\begin{array}{l}\text { EOG ratio in } \\
\text { affected eye } \\
(\%)\end{array}$ & $\begin{array}{l}\text { EOG ratio in } \\
\text { unaffected eye } \\
(\%)\end{array}$ \\
Subject index & 86 & 255 \\
\hline $10^{\star}$ & 95 & 238 \\
24 & 100 & 301 \\
1 & 100 & 346 \\
$2^{\star}$ & 100 & 316 \\
$6^{\star}$ & 100 & 249 \\
4 & 100 & 262 \\
20 & 100 & 300 \\
$27^{\star}$ & 100 & 243 \\
$17^{\star}$ & 100 & 250 \\
$26^{\star}$ & 100 & 275 \\
13 & 100 & 301 \\
11 & 100 & 227 \\
$16^{\star}$ & 110 & 250 \\
7 & 117 & 264 \\
15 & 120 & 264 \\
5 & 129 & 274 \\
$25^{\star}$ & 145 & 223 \\
9 & 146 & 219 \\
8 & 148 & 205 \\
19 & 177 & 216 \\
23 & 188 & 250 \\
12 & 189 & 190 \\
21 & 200 & 220 \\
3 & 208 & 244 \\
14 & 227 & 241 \\
28 & 300 & 424 \\
18 & 382 & 363 \\
22 & 145 & 265 \\
$M$ Mean & 68 & 50 \\
SD & & \\
& &
\end{tabular}

$\star$ Rubeosis iridis

trough, Dt) and the maximum EOG value during light (light peak, Lp). The whole procedure lasted approximately 35 minutes. The head position, eye movements, and pupil diameter of the subject were monitored using an infrared closed circuit TV camera.

All results were analysed using two-tailed paired or unpaired $t$ tests, as appropriate.

\section{Results}

Twenty eight patients were included in the study. Patients underwent electro-oculography between 14 and 48 days from onset of CRVO (mean 32 (SD 8) days). Eight patients (29\%) developed rubeosis iridis during a 9 month
Figure 1 The EOG Lp/Dt ratio in the affected and unaffected eyes of patients with acute $C R V O(\star$. rubeosis iridis).

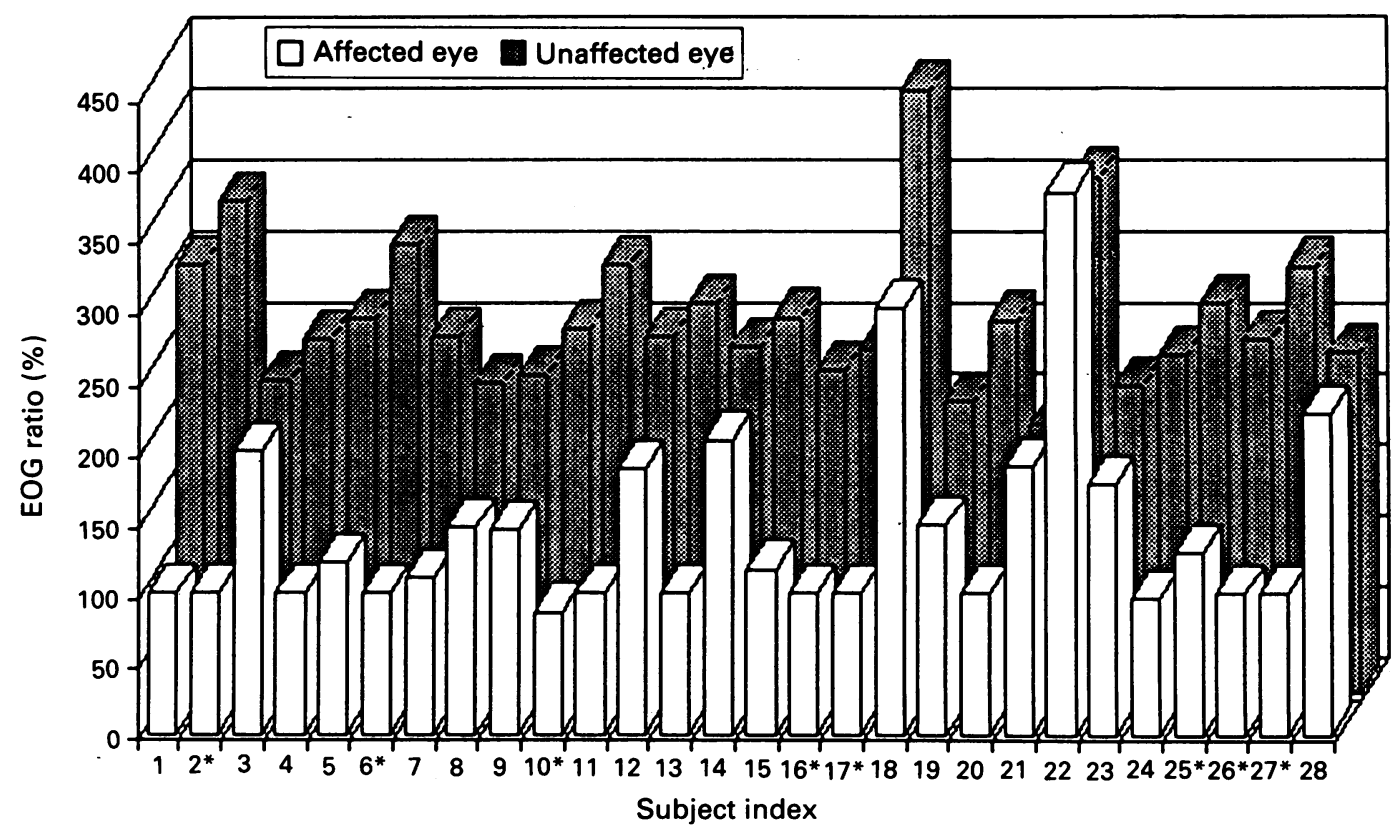




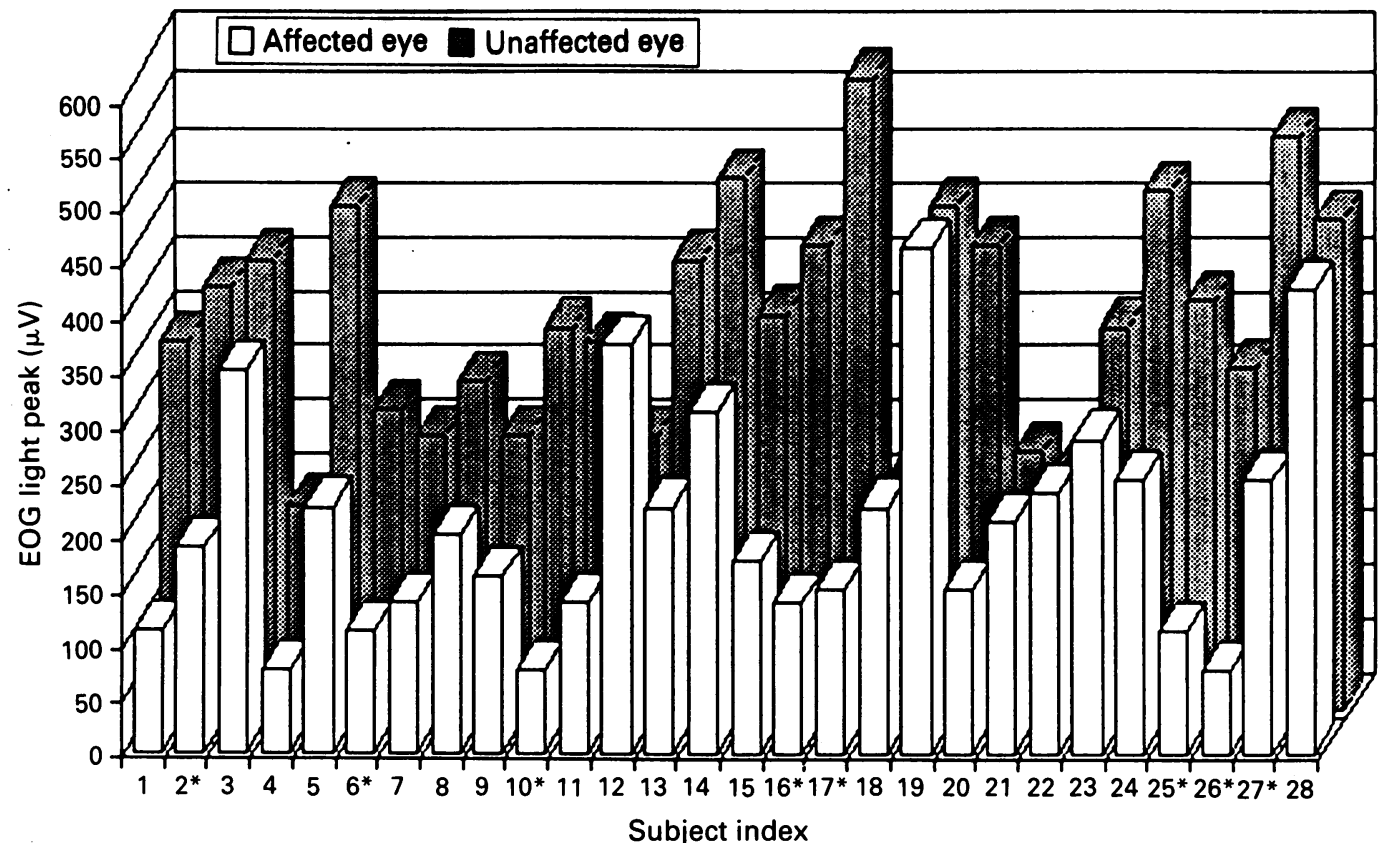

Figure 2 The EOG Lp amplitude in the affected and unaffected eyes of patients with acute'CRVO ( ${ }^{\star}$ : rubeosis iridis).

follow-up period. The rubeosis began at the pupil margin in six of these eight patients, on the trabecular meshwork alone in one patient, and at both of these sites in the remaining patient.

The EOG ratio $(\mathrm{Lp} / \mathrm{Dt}) \times 100$ was smaller in the affected than in the unaffected eye of all but one patient (Fig 1). The range of the EOG ratios for the unaffected eyes was $190-424 \%$, and $86-$ $382 \%$ for the affected eyes (Table 1). In 20 patients $(71 \%)$ the EOG ratio of the affected eye was less than $170 \%$. An EOG Lp/Dt ratio of less than $170 \%$ is considered abnormal in our laboratory and in others. ${ }^{211}$

The mean value of the EOG ratio of all affected eyes was 146\% (SD 68\%), and of all unaffected eyes $265 \%$ (SD 50\%). This difference is statistically significant $(\mathrm{p}<0.001)$. Table 1 shows the EOG ratios of all patients, ranked in ascending order by the affected eye to facilitate interpretation of the data.

The EOG Lp/Dt ratio can be affected by changes in either the $\mathrm{Lp}$ or Dt. The mean $\mathrm{Lp}$ amplitude of affected eyes (208 (SD 101) $\mu \mathrm{V}$ )

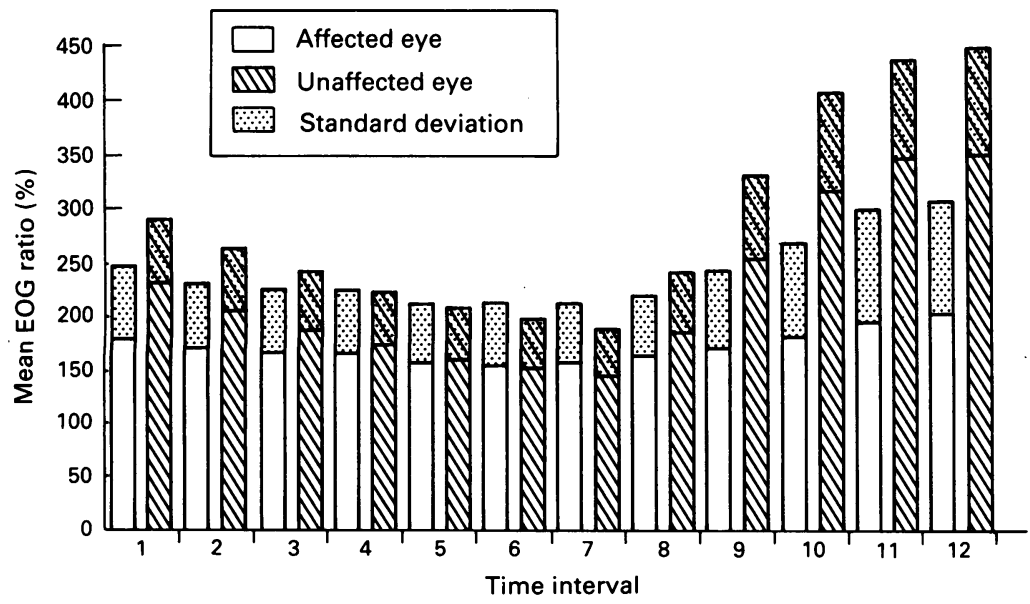

Figure 3 The mean and standard deviation of all patients' EOG Lp/Dt ratios in each time interval of 2 minutes. Time intervals $1-6$ were during dark and time intervals $7-12$ were during light $(n=28)$. It can be seen that the largest differences between affected and unaffected eyes occurred in the light (time intervals 11 and 12), and that at the end of the dark period (time interval 6) there were no differences between affected and unaffected eyes. was significantly smaller $(\mathrm{p}<0.001)$ than that of unaffected eyes (361 (SD 100) $\mu \mathrm{V})$. This is illustrated in Figures 2 and 3 (particularly in time intervals 11 and 12). The difference between the mean Dt amplitude of affected eyes (150 (SD 60) $\mu \mathrm{V}$ ) and unaffected eyes (142 (SD 47) $\mu \mathrm{V}$ ) was not statistically significant. This is shown in Figure 3 (time intervals 6 and 7).

To facilitate comparisons the amplitude of the Lp of the affected (A) eye was expresssed as a percentage ratio of that of the unaffected (NA) eye and the patients were ranked in ascending order according the value of this ratio (A/ NA) $\times 100$ (Table 2). Fourteen patients had an A/NA ratio of less than $50 \%$. It can be seen from

Table $2 L P$ amplitude $(\mu V)$ of the affected $(A)$ eye, expressed as percentage ratio of that of the unaffected (NA) eye. The patients are ranked in ascending order according to the value of this ratio $100 \mathrm{~A} / \mathrm{NA}$

\begin{tabular}{llll}
\hline $\begin{array}{l}\text { Subject } \\
\text { index }\end{array}$ & $\begin{array}{l}\text { EOG light peak } \\
\text { in affected } \\
\text { eye }(A)\end{array}$ & $\begin{array}{l}\text { EOG light peak } \\
\text { in unaffected } \\
\text { eye }(N A)\end{array}$ & $\begin{array}{l}100 \\
A / N A \\
(\%)\end{array}$ \\
\hline $10^{\star}$ & 75 & 350 & \\
$26^{\star}$ & 75 & 312 & 21 \\
$17^{\star}$ & 150 & 575 & 24 \\
$25^{\star}$ & 112 & 375 & 26 \\
$16^{\star}$ & 137 & 425 & 30 \\
1 & 112 & 337 & 32 \\
20 & 150 & 425 & 33 \\
4 & 75 & 187 & 35 \\
11 & 137 & 337 & 40 \\
$6^{\star}$ & 112 & 275 & 41 \\
$27^{\star}$ & 250 & 525 & 41 \\
$22^{\star}$ & 187 & 387 & 48 \\
15 & 175 & 362 & 48 \\
5 & 225 & 462 & 48 \\
24 & 250 & 475 & 49 \\
13 & 225 & 412 & 53 \\
7 & 137 & 250 & 55 \\
14 & 312 & 487 & 64 \\
9 & 162 & 250 & 65 \\
8 & 200 & 300 & 67 \\
23 & 287 & 350 & 82 \\
3 & 350 & 412 & 85 \\
21 & 212 & 237 & 99 \\
28 & 425 & 450 & 100 \\
19 & 462 & 462 & 105 \\
22 & 237 & 225 & 106 \\
18 & 225 & 212 & 150 \\
12 & 375 & 250 & \\
Mean & 208 & 361 & \\
SD & 101 & 100 & \\
\hline & & & \\
\hline & & &
\end{tabular}

$\star$ Rubeosis iridis 
Table 2 that all the eight patients who developed rubeosis iridis had an $A / N A L p$ ratio at presentation of $48 \%$ or less, and that no patient developed rubeosis whose $\mathrm{A} / \mathrm{NA} \mathrm{Lp}$ ratio at presentation was greater than $48 \%$.

When patients with EOG Lp/Dt ratios above $170 \%$ were compared with those below $170 \%$, there were no statistically significant differences in age, sex, or time from CRVO onset to EOG testing, between the two groups. Nor were there any statistically significant differences in these parameters between those patients with an A/NA $L p$ ratio above $48 \%$ and those with an $A / N A L p$ ratio of $48 \%$ or below.

\section{Discussion}

The results presented above show that in this prospective masked study the EOG $\mathrm{Lp} / \mathrm{Dt}$ ratio is significantly reduced in the affected eyes of a group of patients with CRVO compared with the unaffected fellow eyes. Further to this group effect the EOG Lp/Dt ratio was in the range that is considered abnormal (less than $170 \%^{12811}$ ) in the affected eye of $71 \%$ of these 28 individuals, whereas that in the fellow eye was $190 \%$ or more in all cases. The EOG $\mathrm{Lp} / \mathrm{Dt}$ ratio in the affected eye of the remaining $29 \%$ of cases was within the normal range in absolute terms, with minimal differences between the two eyes. This may partly explain the negative findings of previous studies. ${ }^{19}$ It has been suggested that recovery of initial EOG abnormalities in patients with CRVO may occur later in the natural history of the condition. ${ }^{910}$ This too may explain the differences between the findings of this study and of others, for EOG measurements in this study were made at the onset of the condition.

The results show that the effects upon the EOG $\mathrm{Lp} / \mathrm{Dt}$ ratio are largely due to a reduction in the amplitude of the light peak (Lp). To explain why CRVO should lead to a reduction in the amplitude of the $\mathrm{Lp}$, the nature of the damage in CRVO should be considered in the light of current understanding of the processes underlying the development of the Lp.

The blood circulation of the inner retinal layers is by the central retinal artery and the central retinal vein. ${ }^{12}$ The RPE, photoreceptors, and outer layers of the neuroretina are supplied by diffusion of oxygen from the choroidal circulation. The watershed between the two circulations is at the level of the bipolar cell dendrites, at the junction of the outer and middle thirds of the outer plexiform layer of the retina. ${ }^{12}{ }^{13}$ It is thought that CRVO leads to ischaemia of the inner retina owing to reduced perfusion consequent upon the outflow obstruction. ${ }^{14}$

The dependence of the $L p$ on a normally functioning neuroretina has been repeatedly proposed from experimental evidence, ${ }^{15-19}$ and it has been suggested that structures within the neuroretina contribute to the development of the $\mathrm{Lp}$, in addition to the photoreceptor/RPE complex. The Dt is believed to reflect the function of the RPE alone ${ }^{8}$ and so impaired retinal circulation would not be expected to produce defects in the Dt. Moreover, it has been noted in animal experiments that the EOG Dt is not affected by central retinal artery occlusion. ${ }^{20}$
That CRVO has been found to affect the Lp implies either involvement of inner elements of the neuroretina in the development of the $L p$, or extension of the damage consequent upon CRVO to involve the RPE and other structures supplied by the choroidal circulation.

Modulation of the standing potential of the RPE is thought to be initiated by chemical factors, but there is debate whether these modulating chemicals are released from the photoreceptors ${ }^{2}$ (supplied by the choroidal circulation) or from more internal retinal structures ${ }^{15-20}$ (supplied by the retinal circulation).

When the amplitude of the $L p$ in the affected (A) and unaffected (NA) eyes were compared by expressing these values as a percentage ratio A/NA, it was found that the ratio ranged from 21-150\% (Table 2). When these values were ranked in ascending order it was observed that only those patients whose ratio was $48 \%$ or less developed rubeosis. In other words, if the amplitude of the Lp of the EOG in the affected eye is less than half of that of the unaffected eye, then there is an increased probability that rubeosis will develop. When the EOG $\mathrm{Lp} / \mathrm{Dt}$ ratio was similarly ranked, it was not as effective as the A/NA ratio in predicting rubeosis (Table 1 ). This may be due to an effect upon the EOG of the across-subject variability in the Dt ratio.

It is important to determine that in those patients with an A/NA ratio of $48 \%$ or below, or those with an EOG Lp/Dt ratio below $170 \%$, the observed increased incidence of rubeosis was not due to other factors. These populations were therefore examined with respect to age, sex and time between CRVO onset and EOG testing. They were compared with their fellow populations of patients whose A/NA ratio was above $48 \%$, and with those whose EOG Lp/Dt ratios were above $170 \%$, respectively. No statistically significant differences were found between the two sets of populations.

Whatever the case, the results appear to be clinically relevant because the degree of EOG abnormality bears some relation to the development of rubeosis iridis. (Rubeosis may also be caused by other ischaemic conditions such as diabetes mellitus and carotid insufficiency.) CRVO is a common cause of loss of vision and may lead to rubeosis (in $29 \%$ of patients in this series), and to potentially painful neovascular glaucoma in $8-25 \%$ of patients. ${ }^{21}$ The risk of these complications, which are more common in ischaemic CRVO, may be significantly reduced by adequate PRP. ${ }^{21-24}$ However, because of the side effects of PRP (including constriction of the visual fields in these patients, who often have poor central vision), this treatment is only indicated in ischaemic cases where the risk of rubeosis is high. Despite this, it has been reported that up to $80 \%$ of patients with CRVO are treated with PRP. ${ }^{24} \mathrm{~A}$ major clinical problem in CRVO is the early identification of high risk cases. Attempts have been made to predict rubeosis using electroretinographic parameters ${ }^{3-7}$ but not, as far as we have been able to ascertain, using the EOG. Because no single method of prediction is ideal none has gained wide acceptance in clinical practice.

In this study no patient whose A/NA EOG Lp 
ratio was more than $48 \%$ developed rubeosis. Of the 14 patients whose A/NA Lp ratio was less than $50 \%$, eight developed rubeosis, five of whom were the patients with the smallest of all A/NA L p ratios. However six of these 14 did not develop rubeosis in spite of a low ratio. The data does not explain in what way these six patients are different.

Further work is required to investigate these aspects and the variation of the EOG with time, for the results above represent readings taken at only one point in the natural history of the condition. However the results do show that no patients developed rubeosis if their A/NA ratio was greater than $50 \%$, which may develop into an important finding in the clinical management of CRVO.

\section{Conclusion}

It has been shown that the EOG is affected in many patients with acute CRVO, and that this is due to an effect on the EOG light peak. This may suggest that retinal control of the EOG occurs in the inner retina but a toxic effect cannot be ruled out. The results have been shown to have clinical relevance because patients with the most severely affected EOG most commonly develop rubeosis. Further investigation into EOG changes in the pathological situation of CRVO may aid our understanding of normal and abnormal retinal function.

The authors acknowledge the help and financial assistance of the special trustees of Bristol Eye Hospital, thank Mr C Barber for reading the manuscript, and $\mathrm{Mr}$ W Ayliffe for drafting the original grant application.

1 Babel J, Stangos N, Korol S, Spiritus M. Ocular electrophysiology. Stuttgart: Georg Thieme, 1977: 92-3.

Galloway NR. Ophthalmic electrodiagnosis. 2nd ed. London: Lloyd-Luke, 1981.

3 Sabates R, Hirose T, McMeel JW. Electroretinography in the prognosis and classification of central retinal vein occlusion. prognosis and classification of cent

4 Kaye SB, Harding SP. Early electroretinography in unilateral central vein occlusion as a predictor of rubeosis iridis. Arch Ophthalmol 1988; 106: 353-6.
5 Breton ME, Quinn GE, Keene S, Dahmen JC, Brucker AJ. Electroretinogram parameters at presentation as predictors of rubeosis in central retinal vein occlusion patients. of rubeosis in central retinal
Ophthalmology 1989; 96: 1343-52.

6 Hayreh SS, Klugman MR, Podhajsky P, Kolder HE. Electroretinography in central retinal vein occlusion: correlation of electroretinographic findings with pupillary abnormalities. Graefes Arch Clin Exp Ophthalmol 1989; 227: 549-61.

7 Morrell AJ, Thompson DA, Gibson JM, Kritzinger EE, Drasdo N. Electroretinography as a prognostic factor of neovascularization in CRVO. Eye 1991; 5: 362-8.

8 Arden GB, Barrada A, Kelsey JH. New clinical test of retina function based upon the standing potential of the eye. BrF Ophthalmol 1962; 46: 449-67.

9 Ashworth B. The electro-oculogram in disorders of the retinal circulation. Am f Ophthalmol 1966; 61: 505-8.

10 Täumer R, Rohde N, Wollensak J. Course of disturbance of EOG in retinal vessel occlusions. Graefes Arch Clin Exp Ophthalmol 1982; 219: 29-33.

11 Papakostopoulos D. Clinical electrophysiology of the human visual system. In: Chiarenza CA, Papakostopoulos D, eds: Clinical applications of cerebral evoked potentials in paediatric Clinical applications of cerebral evoked potentials in paec

12 Warwick R. Eyeball. In: Eugene Wolff's anatomy of the eye and orbit. 7th ed. London: H K Lewis, 1976: Chapter II, 145-9.

13 Newell FW. Ophthalmology principles and concepts. 6th ed. St Louis: Mosby, 1986: 31 .

14 Behrman S. Retinal vein obstruction. Brf Ophthalmol 1962 46: $336-42$.

5 Thaler A, Heilig P. EOG and ERG components in ischaemic retinopathy. Ophthalmic Res 1977; 9: 38-46.

16 Kawasaki K, Yamamoto S, Yonemura D. Electrophysiologica approach to clinical test for the retinal pigment epithelium. Acta Soc Ophthalmol fpn 1977; 81: 1303-12.

17 Textorius O, Skoog K, Nilsson SEG. Studies on the acute and late stages of experimental central retinal artery occlusion in the cynomolgus monkey. Acta Ophthalmol 1978; 56: in the cync.

18 Valerton JM, Van Norren D. Intraretinal recordings of slow electrical responses to steady illumination in the monkey: isolation of receptor responses and the origin of the ligh peak. Vis Res 1982; 22: 393-9.

19 Bruinink A, Dawis S, Niemeyer G, Lichtensteiger W. Catecholaminergic binding sites in cat retina, pigment epithe lium and choroid. Exp Eye Res 1986; 43: 147-51.

20 Gouras P, Carr RE. Light-induced DC responses of monkey retina before and after central retinal artery interruption. Invest Ophthalmol 1965; 4: 310-7.

21 Sanborn GE, Margaral LE, Jaeger EA. Venous occlusive disease of the retina. In: Tasman W, Jaeger EA, eds. Duane's clinical ophthalmology. Philadelphia: Lippincott, 1989: vol 3; Chapter 15. 11.

22 Laatikainen L. Preliminary report of retinal panphotocoagulation on rubeosis iridis and neovascular glaucoma. tion on rubeosis iridis and

23 Margaral LE, Brown GC, Augsburger JJ, Donoso LA Efficacy of panretinal photocoagulation in preventing neovascular glaucoma following central retinal vein occlusion. vascular glaucoma following cen
Ophthalmology 1982; 89: 780-4.

24 Laatikainen L. A prospective follow-up study of panretinal photocoagulation in preventing neovascular glaucoma folphotocoagulation in preventing neovascular glaucoma folClin Exp Ophthalmol 1983; 220: 236-9. 